\title{
Obstacle Avoidance Method for Electric Wheelchairs Based on a Multi-Layered Non-Contact Impedance Model
}

\author{
Haruna Kokubo, Taro Shibanoki \\ 4-12-1 Nakanarusawacho, Hitachi, 316-8511, Ibaraki University, Japan \\ 16nm712n@vc.ibaraki.ac.jp,taro.shibanoki.ts@vc.ibaraki.ac.jp \\ http://bs.cis.ibaraki.ac.jp/ \\ Takaaki Chin \\ 1070 Akebonocho, Nishi-Ku, Kobe, 651-2181, Hyogo Rehabilitation Center, Japan \\ t-chin@pure.ne.jp \\ Toshio Tsuji \\ 1-3-2 Kagamiyama, Higashi-Hiroshima, 739-8511, Hiroshima University, Japan \\ tsuji@bsys.hiroshima-u.ac.jp
}

\begin{abstract}
This paper proposes an obstacle avoidance method based on a multi-layered non-contact impedance model for control of the biosignal-based electric wheelchair. The proposed system can calculate a virtual repulsive force before the collision by multi-layered impedance fields covered around it. This system therefore regulates desired path to avoid obstacles in a variety of situations. In the experiments, the mobile robot passed through obstacles smoothly, and could stop emergently to avoid the obstacle in front of the robot owing to virtual forces calculated by the proposed model.
\end{abstract}

Keywords: obstacle avoidance, non-contact impedance, electric wheelchairs, people with disabilities

\section{Introduction}

According to the World Health Organization (WHO), the population of people with disabilities worldwide accounts for almost $15 \%$ and is gradually increasing [1]. Electric wheelchairs and other mobility aids are used to support disabled people in independent living by compensating for lost function. In particular, an electric wheelchair that can be controlled with biosignals such as electroencephalograms (EEGs) and electromyograms (EMGs) has recently attracted attention [2] - [4]. The authors' research group also developed the Cybernetic Human Robot Interface System (CHRIS), which can be used to operate wheelchairs, domestic appliances, video games and other equipment using biosignals [4]. The system has a number of distinctive features, such as adaptability to input signal variations and various input types in line with user requirements. However, these systems can malfunction due to biosignal fluctuations associated with unintended motions. It is also necessary to provide a motion assistance method with these approaches, as obstacle avoidance depends on the operator.

A number of conventional techniques are applied to determine appropriate paths for distinction based on artificial potential fields and fuzzy logic using neural networks and other methods [5], [6]. These systems allow robots to avoid obstacles based on virtual force and so on, but tend to focus only on maintaining the minimum distance necessary for avoidance. The application of such technology to biosignal-controlled electric wheelchairs requires consideration of how to achieve natural movement rather than simple obstacle avoidance.

In this regard, a method known as non-contact impedance control supports natural collision prevention [7] - [9]. The technique can be used to generate virtual external forces that act on the robot based on virtual impedance between the robot and obstacles. (including other robots), which allows the robot to steer away from anything in its path. However, this requires the setting of appropriate parameters for various situations. Additionally, as such approaches are premised on the control of mobile robots, it is necessary to develop a natural obstacle-avoidance method for biosignalcontrolled electric wheelchairs.

This paper proposes an obstacle avoidance system for electric wheelchairs based on a non-contact impedance model. The system helps to prevent collisions using mechanical impedance-based covered virtual walls around the robot. It supports smooth avoidance of obstacles and enables collision-preventing emergency stops using virtual walls with different impedance parameters. 


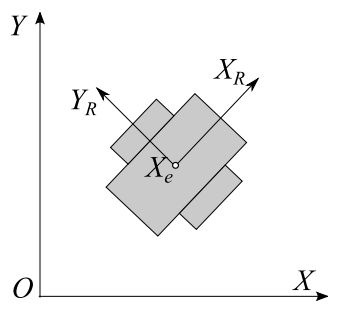

Fig. 1. Kinematics of a mobile robot

\section{Control of the mobile robot based on a Multi- Layered Non-Contact Impedance Model}

In this study, the mobile robot was compressed into a mass point with two translational degrees of freedom (Fig. 1), and motion planning was performed.

The robot can determine the distance between itself and nearby obstacles using an internal sensor. Multiple virtual walls based on mechanical impedance are used to avoid collision with obstacles via calculation of related virtual external forces.

Figure 2 illustrates the concept of the multi-layered non-contact impedance model. It is considered that $P$ obstacles are present in the direction of movement of the robot, which is surrounded by virtual circles (referred to here as virtual walls) with radii of $q_{i}$, $(i=1,2, \ldots, I)$ based on a mechanical impedance model.

When the obstacle comes within the $i$ th virtual wall, the norm vector from the wall's surface to the obstacle $d \boldsymbol{X}_{o}^{i} \in \mathfrak{R}^{2}$ is represented as

$$
d \boldsymbol{X}_{o}^{i}=\boldsymbol{X}_{r}-q_{i} \boldsymbol{n} .
$$

Here, $\boldsymbol{X}_{r}$ is the vector of displacement from the center of the sphere (the mobile robot's position) $\boldsymbol{X}_{e} \in \mathfrak{R}^{2}$ to the $p$ th obstacle ${ }^{(p)} \boldsymbol{X}_{o} \in \mathfrak{R}^{2}(p=1,2, \ldots, P)$. The direction vector $\boldsymbol{n}$ reflecting the distance between the margin of the virtual wall and the obstacle $p$ is defined as

$$
\begin{aligned}
& \boldsymbol{X}_{r}=\boldsymbol{X}_{e}-{ }^{(p)} \boldsymbol{X}_{o} . \\
& \boldsymbol{n}=\left\{\begin{aligned}
\frac{\boldsymbol{X}_{r}}{\left\|\boldsymbol{X}_{r}\right\|}, & \left\|\boldsymbol{X}_{r}\right\| \neq 0 \\
0, & \left\|\boldsymbol{X}_{r}\right\|=0
\end{aligned}\right.
\end{aligned}
$$

If the obstacle is inside the virtual wall $\left(q_{i+1}<\right.$ $\left.\left\|\boldsymbol{X}_{r}\right\| \leq q_{i}\right)$, the virtual impedance between the robot and the obstacle causes exertion of a virtual external force on the robot:

$\boldsymbol{F}_{o}^{i}$

$$
=\left\{\begin{array}{cl}
\boldsymbol{M}_{i} d \ddot{\boldsymbol{X}}_{o}^{\iota}+\boldsymbol{B}_{i} d \dot{\boldsymbol{X}}_{o}^{\iota}+\boldsymbol{K}_{i} d \boldsymbol{X}_{o}^{i}, & q_{i+1}<\left\|\boldsymbol{X}_{r}\right\| \leq q_{i} . \\
0, & \text { otherwise }
\end{array} .\right.
$$

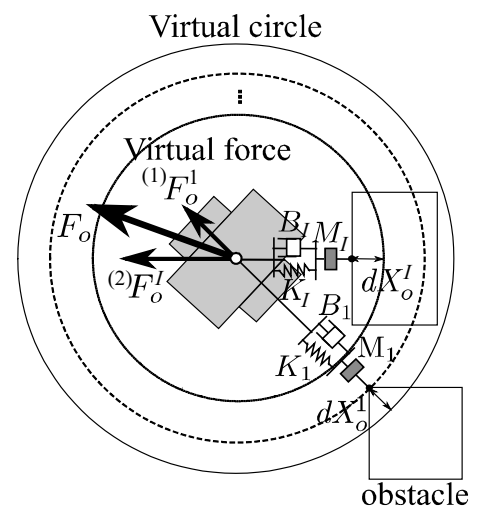

Fig. 2. Structure of the proposed multi-layered non-contact impedance model

Here, $\boldsymbol{M}_{i}, \quad \boldsymbol{B}_{i}$ and $\boldsymbol{K}_{i}$ are the inertia, damping and spring matrices of the non-contact impedance, respectively. It should be noted that if the obstacle is outside the $i$ th virtual wall, the virtual force $\boldsymbol{F}_{o}^{i}$ is zero.

The proposed method supports obstacle avoidance with multi-layered non-contact impedance using various parameters. The virtual extra force from all obstacles in front of the robot is calculated as

$$
\boldsymbol{F}_{o}^{i}=\sum_{p=1}^{P}{ }^{(\boldsymbol{p})} \boldsymbol{F}_{o}^{i} .
$$

Finally, the resultant force acting on the mobile robot in the non-contact impedance model is defined as

$$
\boldsymbol{F}_{o}=\sum_{i=1}^{I} \boldsymbol{F}_{o}^{i} .
$$

Additionally, the dynamic equation for the robot regarding non-contact impedance control is expressed as

$$
\boldsymbol{M}_{e} \Delta \ddot{\boldsymbol{X}}+\boldsymbol{B}_{e} \Delta \dot{\boldsymbol{X}}+\boldsymbol{K}_{e} \Delta \boldsymbol{X}=\boldsymbol{F}_{e}+\boldsymbol{F}_{o} .
$$

Here, $\boldsymbol{M}_{e}, \boldsymbol{B}_{e}$ and $\boldsymbol{K}_{e}$ are the inertia, damping and spring matrices of the robot, respectively.

A displacement vector $\Delta \boldsymbol{X}$ is given using the reference position $\boldsymbol{X}_{t}=\left(x_{t}, y_{t}\right)^{\mathrm{T}}$ and the current position of the robot $\boldsymbol{X}_{e}=\left(x_{e}, y_{e}\right)^{\mathrm{T}}$ based on $\Delta \boldsymbol{X}=\boldsymbol{X}_{t}-\boldsymbol{X}_{e}$. The limitations of $\Delta \boldsymbol{X}$ and $\Delta \ddot{\boldsymbol{X}}$ [6] and the elements $\boldsymbol{K}_{e} \Delta \boldsymbol{X}$ and $\boldsymbol{M}_{e} \Delta \ddot{\boldsymbol{X}}$ in Equation (7) are ignored. Thus, the equation translates to

$$
\boldsymbol{B}_{e} \Delta \dot{\boldsymbol{X}}=\boldsymbol{F}_{e}+\boldsymbol{F}_{o} .
$$

In Equation (8), the velocity increment $\Delta \dot{\boldsymbol{X}}$ is calculated as

$$
\Delta \dot{\boldsymbol{X}}=\frac{1}{\boldsymbol{B}_{e}}\left(\boldsymbol{F}_{e}+\boldsymbol{F}_{o}\right)
$$


Finally, $\dot{\boldsymbol{X}}_{v}$ is defined as the velocity of control that allows the robot to avoid an obstacle using

$$
\dot{\boldsymbol{X}}_{v}=\dot{\boldsymbol{X}}_{e}-\Delta \dot{\boldsymbol{X}}
$$

\section{Simulation Experiment}

\subsection{Experimental Conditions}

To evaluate the efficacy of the proposed method, two simulation experiments were performed with immobile obstacles located (1) randomly and (2) along the path of movement. Simulations confirm whether the robot can emergently stop before collision as well as pass through obstacles using proposed method only.

The robot had an initial position of $(0.0,0.0)[\mathrm{m}]$ and moved toward a target position $(5.0,0.0)[\mathrm{m}]$ with an initial speed of $(0.6,0.0)[\mathrm{m} / \mathrm{s}]$ in experiment $(1)$. In experiment (2), the robot moved in a straight line at a speed of $(0.2,0.0)[\mathrm{m} / \mathrm{s}]$. The parameters used in the simulation were $I=1, q_{1}=0.6 \quad[m], \quad \boldsymbol{M}_{1}=$ $(10.0,10.0) \quad[\mathrm{kg}], \quad \boldsymbol{B}_{1}=(5.0,5.0) \quad[\mathrm{Nm} / \mathrm{s}], \quad \boldsymbol{K}_{1}=$ $(10.0,10.0)[N / s]$ for experiment (1), and $I=2$, $q_{1}=0.6[\mathrm{~m}], \boldsymbol{M}_{1}=(0.21,0.21)[\mathrm{kg}], \boldsymbol{B}_{1}=(0.8,0.8)$ $[\mathrm{Nm} / \mathrm{s}], \boldsymbol{K}_{1}=(1.0,1.0)[\mathrm{N} / \mathrm{m}], q_{2}=0.4[\mathrm{~m}], \boldsymbol{M}_{2}=$ $(16.8,16.8) \quad[\mathrm{kg}], \quad \boldsymbol{B}_{2}=(12.6,12.6) \quad[\mathrm{Nm} / \mathrm{s}], \quad \boldsymbol{K}_{2}=$ $(1000.0,1000.0)[N / s]$ for experiment $(2)$.

\subsection{Results and discussion}

Figures 3 and 4 show the results of the experiments ((a) for (1) and (b) for (2)). Figure 3 shows the paths of the robot in each experiment, and Fig. 4 shows, from top to bottom, velocities (overall and $x$-/y-axis) and virtual forces in each direction under the proposed method.

It can be seen from Fig. 3 (a) that the robot passed straight by two obstacles and altered its path to avoid another on the right. After the avoidance maneuver, the robot corrected its path toward the target position, avoided obstacles on the left and finally arrived at the target position. Figure 4 (a) shows that the robot slowed down due to virtual forces from the presence of two obstacles under the proposed method at around $1.0[\mathrm{~s}]$ before sensing virtual forces from behind and accelerating to avoid an obstacle at 1.8 [s]. The robot adjusted its velocity as appropriate in response to virtual impedance forces indicating the distance from obstacles on each side.
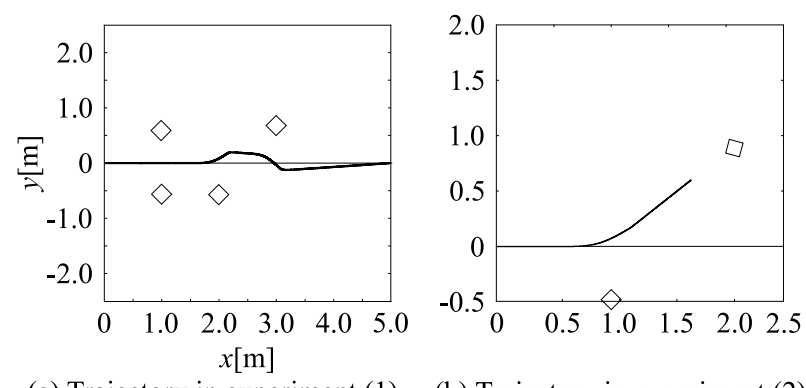

(a) Trajectory in experiment (1)

(b) Trajectory in experiment (2)

Fig. 3. Trajectories of each experiment

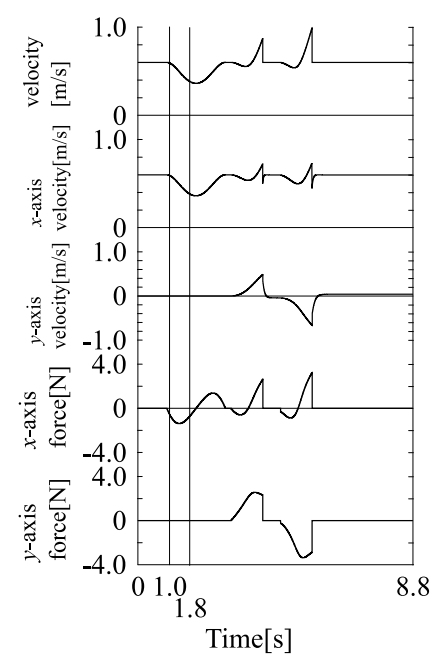

(a) Velocity and virtual force in experiment (1)

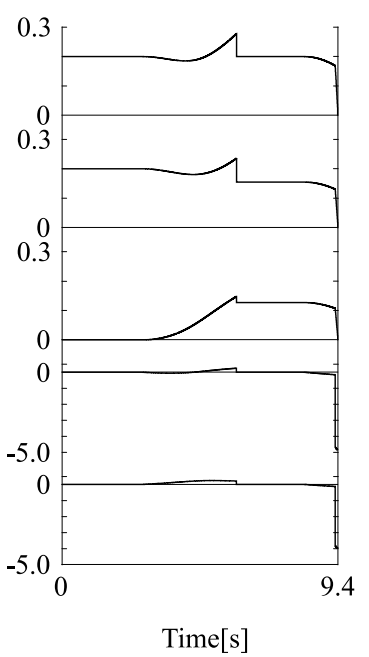

(b) Velocity and virtual force in experiment (2)
Fig. 4. Velocities and virtual forces of each experiment

Figures 3 (b) and 4 (b) show other obstacles in the robot's path after collision avoidance. The robot decelerated gradually, and finally stopped before collision under the influence of virtual forces from the virtual wall with higher stiffness parameters from the obstacle at around $9.4[\mathrm{~s}]$.

The results indicate that the proposed method supports collision avoidance in various situations.

\section{Practical Experiment}

A trajectory-tracking control experiment using an actual mobile robot (iRobot Create 2, iRobot Corp.) was performed with the position coordinates detailed in Section 3. The target electric wheelchairs generally have pairs of propulsion and caster wheels, but a control method for a robot with two independently driven wheels was considered here for simplicity. Sufficient friction between the drive wheels and the floor to avoid wheel spin was assumed. 


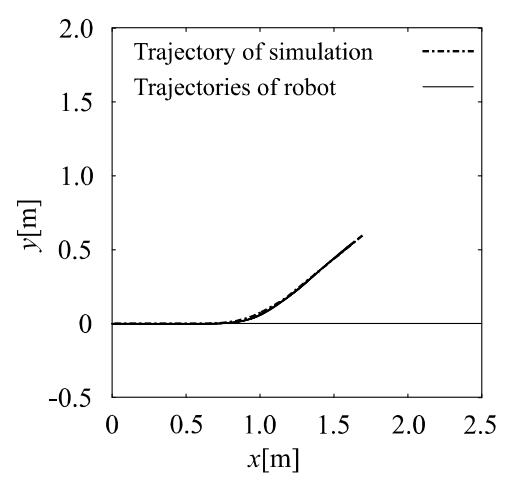

(a) Trajectories of robot
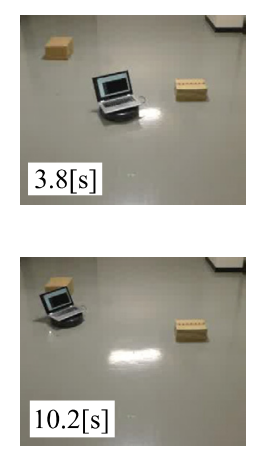

(b) Scenes of robot
Fig. 5. Experimental results for practical experiment

The position coordinates were regarded as target points to be tracked by the robot. Robot position/posture data and target position coordinates were used to determine target velocity, which was defined for each wheel $\left(v_{L}\right.$, $\left.v_{R}\right)^{\mathrm{T}}$ using the distance $L_{T}$ and direction $\theta_{T}$ between the robot and the target position as

$$
\begin{aligned}
& v_{L}=K_{v} \cdot L_{T}+\Delta v, \\
& v_{R}=K_{v} \cdot L_{T}-\Delta v, \\
& \Delta v=K_{t} \theta_{T}+K_{t D} \dot{\theta_{T}} .
\end{aligned}
$$

Here, $K_{v}, K_{t}$ and $K_{t D}$ are pre-set constants.

Figure 5 (a) shows the paths observed in the simulation and practical experiments. Solid lines show the results of 10 trials in the practical experiment. Hardly any difference between the results of the two conditions is observed. Figure 5 (b) shows tracking in the sixth trial. As in the simulation experiment, the robot detected the obstacle and decelerated to pass by it with individual wheel velocity regulation in response to virtual reaction forces with the proposed model. The robot subsequently decelerated to avoid collision with other obstacles along its path in response to alternate contact with outer and inner virtual walls.

These results indicate that the proposed multi-layered non-contact impedance model enables obstacle avoidance in various situations. However, the scope of the tracking experiment was limited to the conditions reported here; no experiment using an actual biosignalcontrolled electric wheelchair was conducted. The authors plan to verify the efficacy of the proposed method with implementation of the model using range sensors.

\section{Conclusion}

This paper proposes a multi-layered non-contact impedance model and its application to obstacle avoidance for electric wheelchairs. In this method, multiple virtual walls based on a mechanical impedance model are located around the robot, enabling natural avoidance of obstacles and emergency stops to prevent collisions. In simulation and practical experiments using the method, the robot avoided obstacles in response to virtual forces based on the model.

In future research, the authors plan to implement the method using distance image sensors and apply it in operation of an actual biosignal-controlled wheelchair [4] to evaluate its effectiveness.

\section{Acknowledgements}

This work was supported by JSPS KAKENHI Grant Number JP26330226.

\section{References}

1. World Health Organization, Disability and health, http://www.who.int/mediacentre/factsheets/fs352/en/, (Accessed November 2016).

2. Y. T. Lin and C. H. Kuo, Development of SSVEP-based Intelligent Wheelchair Brain Computer Interface Assisted by Reactive Obstacle Avoidance, in Proc. 2016 IEEE International Conference on Industrial Technology, (Saratov, Russia, 2016), pp. 1572-1577.

3. K.Tanaka, K.Matsunaga and H. O. Wang, Electroencephalogram-based Control of an Electric Wheelchair, IEEE Trans. on Robotics 21(4) (2005) 762-766.

4. K. Shima, R. Eguchi, K.Shiba and T.Tsuji, CHRIS: Cybernetic Human-Robot Interface Systems, in Proc. 36th International Symposium on Robotics, (2005) WE1C3

5. M. Massari, G. Giardini and F. B-Zazzera, Autonomous Navigation System for Planetary Exploration Rover Based on Artificial Potential Fields, in Proc. Dynamics and Control of Systems and Structures in Space 6th Conference, (Riomaggiore, Italy, 2004), pp. 153-162.

6. R. M. F. Alves and C. R. Lopes, Obstacle avoidance for mobile robots: a Hybrid Intelligent System Based on Fuzzy Logic and Artificial Neural Network, in Proc. 2016 IEEE International Conference on Fuzzy Systems, (Vancouver, Canada2016), pp. 1038-1043.

7. T. Thsuji, M.Hatagi and M. Kaneko, Non-Contact Impedance Control for Manipulators, J. the Robotics Society of Japan 15(4) (1997) 616-623.

8. E. S. Jang, S.Jung and T. C. Hsia, Collision Avoidance of a Mobile Robot for Moving Obstacles Based on Impedance Force Control Algorithm, in Proc. 2005 IEEE/RSJ International Conference on Intelligent Robots and Systems, (Edmonton, Canada, 2005), pp.383-387.

9. Z. Li, Z.Wu and Y. Fu, Dynamic Obstacle Avoidance of Mobile Robot Tele-operation Based on Non-contact Impedance Control, in Proc. 2014 11th World Congress on Intelligent Control and Automation, (Tianyou, China, 2014), pp. 1421-1426. 